\title{
Ergebnisse der Kriegserfahrungen für die Physiologie der Ernährung und für die Diätetik.
}

Von A. Loewy und H. Strauss.

\section{II.}

\section{Ergebnisse fiur die praktische Diätetik. Von Prof. H. Strauss.}

Während es den Fortschritten der Hygiene und der ausgezeichneten Organisation unserer Behörden gelungen ist, die Heimsuchung unserer Bevölkerung durch Seuchen, die früher besonders schlimme Folgen längerer Kriege darstellten, auf ein in Anbetracht der in Frage kommenden Verhältnisse geradezu erstaunlich niedriges Maß herabzudrücken, konnte es uns doch nicht gelingen, die Folgen einer mehr als vier Jahre langen, in erbarmungsloser Weise durchgeführten Hungerblockade auf die Bevölkerung des Heimatgebietes auf einen mit der Gesundheit auch nur einigermaßen verträglichen Grad zu mildern. Dies gilt nicht nur für Gesunde, sondern in weit höherem Grade noch für Kranke und für Schwache. Zahllos sind bei diesen die Opfer gewesen, welche lediglich als die Folge mangelnder Ernährungsmöglichkeiten dahingerafft worden sind. In besonderem Grade ist dabei die Bedeutung der für bestimmte Krankheiten unzureichenden Ernährungsweisen zutagegetreten, und wir mußten leider eine Reihe von Erfahrungen machen, die als Beiträge für die Klinik und zum Teil auch für die praktische Diätetik einer besonderen Betrachtung wert erscheinen. Diese Erfahrungen beziehen sich sowohl auf quantitative, als auch auf qualita tive Fragen der Ernährung. Soweit die ersteren in Betracht kommen, ist es zunächst Sache des Physiologen, die Folgen der chronischen Unterernährung auf den Gesunden im einzelnen zu studieren und zu erörtern. Den Kliniker interessierte die Frage der Unterernährung erst von dem Moment ab, wo sie sich als krankmachender Faktor bemerkbar machte. Er schenkte deshalb dem ersten Stadium der Unterernährung, d. h. dem Stadium der Gewichtsabnahme ohne krankhafte Nebenerscheinungen, weniger Beachtung als dem zweiten Stadium, d. h. dem Stadium der Gewichtsabnahme mit krankhaften, aber n och nicht gefährlichen Nebenerscheinungen, und dem dritten 
Stadium, in welchem die Nebenerscheinungen schon einen lebensdrohenden Charakter angenommen hatten.

Das erste Stadium hatte allerdings auch für den Kliniker oft insofern ein.besonderes Interesse, als es zuweilen auffällig war, wie lange es bei Personen, die nur von rationierter Nahrung lebten, dauerte, bis klinisch sichtbare Folgen der Unterernährung zutagegetreten sind, und insofern, als es oft von besonderem Interesse war, $\mathrm{zu}$ sehen, wie verschieden sich die Größe und der Gang der Gewichtsabnahme bei den einzelnen Fällen bemerkbar machten. Man konnte sich dabei gar oft von der Tatsache überzeugen, daß Fragen des Stoffwechsels in der Klinik vielfach doch weit komplizierter liegen, als es nach den meist in präzisen Formeln dargestellten Ergebnissen der Physiologie des Stoffwechsels der Fall zu sein scheint. Sahen wir doch bei den einzelnen Personen beträchtliche Unterschiede in Grad und Gang der Gewichtsabnahme nicht bloß entsprechend der Verschiedenheit in der Art der Anpassung an die neuen Verhältnisse auftreten. Die Beobachtung, daß neben Personen, welche rasch abnahmen und bald verfielen, andere trotz kärglicher Ernährung ihren Pannikulus doch auffällig lange verteidigen konnten, läßt sich wohl am besten mit einer individuellen Verschiedenheit in der Wirkung derjenigen endokrinen Vorgänge erklären, welche als Blasebalg auf die Verbrennungsprozesse des menschlichen Körpers einwirken. Die genannte Beobachtung, die jedenfalls durch die Differenz zwischen Ruhe und Bewegung in den einzelnen Fällen nicht immer ausreichend erklärt werden konnte, läßt sich übrigens gut in Uebereinstimmung mit einer alten klinischen Erfahrung bringen, nach welcher Mast- und Entfettungskuren nicht immer nach einem einfachen kalorischen Rechenexempel verlaufen, sondern oft von unbekannten „,konstitutionellen" bzw. „endokrinen" Faktoren beeinflußt werden. Haben wir doch schon früher die Fälle von ,endokriner" Fettsucht von den Fällen von Mast- und Sparfettsucht zu scheiden gelernt und weiterhin auch die Erfahrung gemacht, daß es Fälle von ,,konstitutioneller" Magerkeit gibt, bei welchen trotz regelrechter Ueberernährung ein entsprechender Gewichtsansatz nicht zu erzielen ist. Im ersten Stadium der Unterernährung fiel zuweilen aber auch noch ein Weiteres auf, insofern, als bei manchen Personen sogar eine Steigerung des Wohlbefindens und - wie wir alsbald erörtern werden - zuweilen sogar ein günstiger Einfluß auf bestimmte Krankheiten festzustellen war. Die erstere ist wohl auf eine allgemeine „Deplethorisierung" bzw. Entlastung der-Zirkulation durch Fettabgabe zurückzuführen. Hat doch die Kriegserfahrung u. a. uns auch gezeigt, wie viel Luxusernährung früher getrieben worden ist. Von Krankheiten sind leichte Fälle von Diabetes sowie zahlreiche Fälle von Gicht vom ersten Stadium der Unterernährung günstig beeinflußt worden. Schon bei der Belagerung von Paris hatte seinerzeit, Bouchardat bei Diabetikern einen günstigen Einfluß der Unterernährung auf den Verlauf des Diabetes festgestellt, und wir haben in den letzten vier Jahren immer und immer wieder beobachten können, daß leichte und mittelschwere Fälle von Diabetes von der allgemeinen Unterernähcung oft sehr günstig beeinflußt worden sind. In den Krankenhäusern und in den ärztlichen Sprechstunden erschienen jedenfalls Diabetiker an sich seltener als früher, und Patienten, über deren Zuckerausscheidung wir von früher her orientiert waren, zeigten eine sichtliche Verminderung oder ein Verschwinden der letzteren. Auch Fälle von Gicht sind während der Kriegszeit weit seltener zur Beobachtung gelangt als während der Friedenszeit. Schwere Diabetiker wurden allerdings von der Unterernährung oft sehr ungünstig beeinflußt, und bei vielen Patienten aus dieser Gruppe hat die mangelnde Ernährungsmöglichkeit den Ablauf der Krankheit entschieden beschleunigt. Auch sehr vorgeschrittene Fälle von Gicht mit chronisch deformierenden Prozessen litten oft sehr unter den Ernährungsschwierigkeiten. Soweit der Diabetes in Frage kommt, stützen die gemachten Erfahrungen die Auffassung derjenigen Autoren, welche die Ursache des Diabetes nicht bloß in einer Störung des Kohlehydratstoffwechsels, - sondern vielmehr in einer Störung des Gesamtst offwechsels sehen. Insbesondere sind sie geeignet, Anschauungen $\mathrm{zu}$ stützen, wie sie Kolisch u. a. im Sinne einer „Reiztheorie" geäußert haben, da es naheliegt, auch hier an eine durch Schonung erzeugte Erregungsverminderung der Funktion endokriner Drüsen zu denken. Jedenfalls verdienen die im Kriege gemachten Beobachtungen über die Bedeutung des ,Maßhaltens im ganzen" auch für die künftige Diabetesbehandlung vollste Beachtung. Soweit die Gicht in Frage kommt, so bedurfte die Berechtigung dieses Satzes allerdings nicht mehr neuer Beweise, trotzdem der günstige Einfluß der Kriegsernährung zum Teil we igstens in der Fleischarmut der Kriegsernährung zu suchen sein dürfte. Freilich hat der günstige Einfluß der systematischen Unterernährung auch bei den leichteren Formen von Zucker- und Gichtkrankheit eine Grenze erkennen lassen. Denn gar mancher Fall auch aus dieser Gruppe ist durch eine zu weitgehende Unterernährung empfindlich geschädigt worden.

Von Interesse war die erzwungene Unternährung u. a. auch für den Geburtshelfer, und zwar mit Rücksicht auf die seinerzeit von Prochownik eingeleiteten Bestrebungen, den Fötus durch knappe Ernährung der Mutter klein zu gestalten. Soweit ich aus persönlichen Mitteilungen von Geburtshelfern erfahren habe, hat es sich aber gezeigt, daß die Größe des Fötus durch die Unterernährung wenig oder gar nicht beeinflußt wird. Der Fötus reißt als Parasit das für ihn notwendige Nahrungsquantum bedingungslos an sich und läßt sich durch den Ernährungszustand der Mutter nur wenig beeinflussen.

In dem bei dem einen früher, bei dem anderen später beginnenden $\mathrm{z}$ weiten Stadium der Unterernährung zeigten sich die Folgen der Unterernährung vor allem von seiten des Nervensystems, und es bestätigte auch die Kriegsernährung die früheren Erfahrungen über die engen Beziehungen zwischen Ernährungszustand und Nervenleistung. Minderung bzw. abnorm rasches Versagen der körperlichen und geistigen Kräfte und erregbares, mürrisches Wesen zeigten sich meist als die ersten Folgen, und es schien oft, als ob das Nachlassen der körperlichen und geistigen Spannkraft nicht bloß durch die Unterernährung allein, sondern zum Teil auch durch die mangelhafte Magenfüllung, allso durch die Verbindung der Unterernährung mit einem dauernden quälenden Hungergefühl erzeugt war. Jedenfalls war nicht selten $\mathrm{zu}$ beobachten, daß schon eine ausreichende Magenfüllung allein geeignet war, einen Teil der Unlustgefühle und der Leistungsverminderung $\mathrm{zu}$ beseitigen. Wir glauben sogar annehmen zu dürfen, daß die hier skizzierte Veränderung der Psyche an der Entstehung des zurzeit in so betrübendem Maße zutage tretenden Widerwillens gegen Arbeit und der allgemeinen Auflehnungsbereitschaft mitbeteiligt ist. In den Erscheinungen selbst sehen wir aber auch nicht etwas Neues, da wir die genannte Steigerung der allgemeinen Erregbarkeit des Nervensystems mit Verminderung der körperlichen und geistigen Leistungsfähigkeit ja nicht selten auch schon früher bei raschen oder auch bei langsam, aber in ten siv durchgeführten Entfettungskuren zu sehen bekommen haben. Die Folgen der Unterernährung haben sieh in evidenter Weise aber auch noch in der Form einer Erhöhung der Krankheitsbereitschaft gegenüber von In fektionen gezeigt. Dies gilt nicht bloß in bezug auf die Tuberkulose, die in der Kriegszeit in erschreckender Weise zugenommen hat, sondern auch in bezug auf gewisse akute Infektionskrankheiten, so besonders auf die Ruhr, die namentlich bei Personen über 50 Jahren im Kriege viel schwerer verlief, als wir es in den Zeiten besserer Ernährung zu sehen gewohnt waren. Auch die hohe Morbidität und Mortalität der Grippe dürfte von der Ernährungslage nicht unbeeinflußt gewesen sein. In manchen Fällen, und dies gilt nicht bloß für die hier genannten Krankheitsgruppen, machten sich die unangenehmen und zuweilen direkt deletären Folgen der Unterernährung besonders in der Rekonvaleszenz bemerkbar, weil es in der Mehrzahl der Fälle nicht möglich war, die für das Rekonvaleszenzstadium zur allgemeinen Kräftigung notwendige Menge und Art der Nahrung zu gewinnen. Verzögerte Erholung trat jedenfalls in der letzten Influenzaepidemie in noch stärkerem Grade zutage, als wir sie bei früheren Epidemien und speziell in der großen Epidemie vom Jahre $1890 \mathrm{zu}$ sehen bekamen. Ganz allgemein hat die Bedeutung des Faktors ,Ernährung" als Stärkungs- und Kräftigungsmittel durch die negativen Erfahrungen der 
letzten Jahre eine besonders eindringliche Betonung erfahren.

Ueber die maximalen Grade der Unterernährung, wie sie sich besonders in der sogenannten ,Oedemkrankheit" gezeigt haben, ist eine große Literatur erschienen. Die Entstehung dieser, in sozialpolitisch weniger gut geleiteten Ländern, wie z.B. in Rußland und in Polen, schon frủher und besser als bei uns bekannt gewesenen Krankheit darf einerseits auf eine übermäBige Zufuhr von Wasser und Salzen, anderseits auf eine alimentär bedingte Oedembereitschaft zurückgeführt werden. Man wird wohl nicht fehlgehen, wenn man als wesentliche Ursache der letzteren eine maximale Verminderung lebenswichtiger Nahrungsstoffe ansieht, womit eine gewisse Beziehung zu den kachektischen Oedemen gegeben wird. Ob der Fettmangel dabei (etwa im Sinne einer mangelhaften Gefäßdichtung?) eine besonders wichtige Rolle spielt, läßt sich schwer entscheiden, doch weisen, wie ich schon früher an anderer Stelle erörtert habe, mancherlei Momente auf eine solche Möglichkeit hin. Auffallend ist jedenfalls die Erfahrung; daß Fettzulagen - aber auch ganz allgemein eine Besserung der Ernährung - in zahlreichen Fällen überraschend schnell, zu einem Verschwinden der Hydropsien geführt haben. Nicht minder auffällig ist aber auch, daß rasche Besserung zuweilen auch schon durch absolute körperliche Ruhe erreicht wurde, deren rascher Erfolg nicht bloß durch eine Einschränkung der Stoffwechselvorgänge, sondern zum Teil wohl durch eine Erleichterung bzw. Verbesserung der Herzarbeit zu erklären sein dürfte. Es läßt sich auch darüber diskutieren, ob und inwieweit die von manchen Seiten angestellten Vergleiche mit den ,Nährmehlschäden" der Kinder und mit den „Haferödemen" der Diabetiker zutreffen. Immerhin ist die Art der Ernährung in den zur Erörterung stehenden Fällen nicht in allen zum Vergleich stehenden Punkten dieselbe, und es ist ferner fraglich, ob die Verhältnisse beim Erwachsenen ohne weiteres mit denjenigen beim Kinde in eine Parallele gesetzt werden dürfen. Bei den Haferödemen ist Grad und Dauer der Unterernährung jedenfalls nicht ohne weiteres mit den Verhältnissen vergleichbar, unter welchen die Hungerödeme in die Erscheinung getreten sind. Weiterhin kann bei den letzteren ein Uebermaß in der Kohlehydratzufuhr höchstens in relativer Form, nicht aber in absoluter Form in Frage kommen. Immerhin sind hier eigenartige Kombinationen in den gegenseitigen Magenverhältnissen verschiedener wichtiger Nahrungsstoffe - es wäre hier $u$. a. auch an ein chronisches Kalkdefizit infolge der Reduktion der Milch- und Eierzufuhr zu denken, das auch sonst für die Ernährung nicht gleichgültig geblieben sein dürfte - in Erwägung zu ziehen. Es soll aber diese Frage, mit welcher sich Jansen u. a. ausführlich beschäftigt haben, hier nicht zum Gegenstand einer ausführlichen Erörterung gemacht werden, sondern es soll hier nur erwähnt werden, daß auch von ,Oedemkrankheit ohne Oedeme " in solchen Fällen gesprochen worden ist. bei welchen man starke Polyurie mit gleichzeitigem progressiven Gewebsschwund und mit hochgradiger Austrocknung sämtlicher Gewebe beobachten konnte, indem man sich vorstellte, da $B$ man in den betreffenden Fällen nur anhydropische Erscheinungsweisen der gleichen Vorgänge, d. h. die Folgen einer abnorm reichlichen Wasserund Salzzufuhr wie bei der Oedemkrankheit vor sich habe. Während man aber bei den Fällen von Oedemkrankeit eine ausgesprochene Blutverwässerung feststellen konnte, war es meist aufällig, in wie geringem Grade bei der allgemeinen Unterernährung meist das Blutgewebe selbst geschädigt wurde, da man leichte Građe von Anämie zwar relativ häufig, schwere Formen dieser Krankheit aber doch nur ziemlich selten unter dem Einfluß der Unterernährung zustandekommen sah.

In bezug auf die einzelnen Nährstoffe ist es Sache der Physiologen, die Frage des Eiweißminimums zu erörtern. Für die Klinik und speziell für die Therapie war aber die Erfahrung, wertvoll, daß man bei ausreichendem Kaloriengehalt der ${ }_{i}^{*}$ Nahrung auch mit relativ kleinen Eiweißrationen, d. h. mit etwa $50 \mathrm{~g}$, die körperliche Leistungsfähigkeit für mäßige und mittlere Anstrengungen ziemlich lange ungestört erhalten kann. Es darf von dieser Feststellung vor allem die diätetische Schonungstherapie der Nierenkranken Nutzen ziehen. Ganz allgemein hat sich aber auch gezeigt, daß das fruher übliche Quantum in der Fleischzufuhr nicht bloß ohne Schaden, sondern sogar mit Vorteil für Gesunde und Kranke ganz erheblich verringert werden darf. Anderseits ist aber auch nicht $\mathrm{zu}$ bestreiten, daß gar mancher Gesunde und Kranke unter der durch die Verhältnisse erzwungenen, sehr weitgehenden Verringerung der Zufuhr des Fleisches tatsächlich gelitten hat, indem er auf die Reizwirkung des Fleisches nicht ohne ein Unbehagen und zuweilen sogar nicht ohne eine Minderung seiner körperlichen und seelischen Spannkraft verzichten konnte. Infolgedessen kann die - namentlich von den Vegetariern angeschnittene - Frage, ob da u ernder völliger Verzicht auf das Fleisch für jedermann zweckmäßig ist, nicht unbedingt bejaht werden. Für die Beurteilung dieser Frage sind übrigens die Kriegserfahrungen nicht gerade in besonderem Grade geeignet, weil die für eine ausreichende Ernährung so wichtigen Nahrungsmittel Milch und Eier auch für viele Kranke nur in recht knapper Weise zur Verfügung standen. Soweit das Fett in Frage kommt, so war auch in der Krankenernährung oft die mangelnde Sättigungskraft fettarmer Speisen zu beklagen, und es ist sogar zu erwägen, ob nicht die Fettarmut der Nahrung mehr oder weniger zu der oft beobachteten Sprödigkeit der Haut mit ihrer Neigung zu Dermatosen beigetragen hat. Harnsäuresedimente im Urin sind allerdings während der fleischarmen Ernährung in der Kriegszeit eine große Seltenheit geworden.

Was von den Reizstoffen des Fleisches gesagt worden ist, darf auch von einigen anderen Reizstoffen wiederholt werden. Kaffee-Ersatz und Tee-Ersatz haben uns gezeigt, was die gewohnten Naturprodukte für uns tatsächlich wert waren, und es hat sich ganz allgemein bei den Ersatzstoffen aufs Neue erwiesen, daß gerade die Psychologie der Ernährung nicht bloß beim Kranken, sondern auch beim Gesunden ein ebenso heikles wie wichtiges Gebiet darstellt. Der alte Satz ,Natura non vult decipi" hat sich auch sonst bei der durch das Fehlen zahlreicher gewohnter Stoffe notwendig gewordenen Aenderung in der Zubereitung zahlreicher Speisen in oft recht unangenehmer Weise bemorkbar gemacht. Wir haben dabei nicht bloB gewisse nur vom Ausland erhältliche Gewürzstoffe, sondern auch zahlreiche Geschmackswirkungen im Auge, wie sie beispielsweise mit den durch Inlandserzeugnisse, so z. B. Eier, Milch, Butter usw. möglichen Kompositionen erzielt werden können. Schwere Mißstände haben sich auf diesem Gebiete nicht bloß bei appetitlosen Kranken, sondern auch bei vielen Kranken ergeben, die auf eine zarte Diät angewiesen waren, da gerade die letztere oft ohne eine besondere Schmackhaftigkeit und Abwechslung schwer durchführbar ist, und zwar nicht bloß bei kapriziösen Kranken, sondern auch bei solchen, welche sich opferwillig in weitestgehender Form der Notlage angepaßt hatten. Zwar hat sich gezeigt, daß auch die Krankenernährung unter dem Einfluß der Not vielfach mit einfacheren Mitteln auskommen kann, als man es in einer Zeit des Ueberflusses für mö̀glich gehalten hat, doch haben die durch die Verhältnisse erzwungenen Einschränkungen in vielen Fällen dem ärztlichen Wirken eine höchst bedauerliche Grenze gesetzt.

Besonders empfindlich machten sich diese Grenzen in den oft geradezu unerträglichen Einschränkungen bemerkbar, zu welchen die Krankenernährung da gezwungen war, wo es darauf ankam, für längere Zeit eine von groben $\mathrm{Be}$ standteilen freie, zarte, insbesondere zellulosearme Kost zu beschaffen. Hier hatte sich für die Krankenernährung oft ein geradezu schreiender, durch nichts zu überwindender Notstand ergeben, der auf direktem oder indirektem Wege zahlreiche Opfer gefordert hat. Wir haben hier vor allem die Verdau ungskranken im Auge, und es ist nicht übertrieben, wenn man behauptet, daß, abgesehen von den an zehrenden Krankheiten leidenden Patienten, kaum eine Gruppe von Kranken von der Hungerblockade so schwer betroffen worden ist wie das Heer der Verdauungskranken. Stellte doch die Behandlung dieser Kranken in der zweiten Hälfte der Kriegszeit für den Arzt oft eine Sysiphusarbeit dar, die den seiner Ohnmacht und gleichzeitig seiner Verantwortung bewußten Arzt zuweilen geradezu zur Verzweiflung bringen konnte. Dazu kam noch, daß die Zahl der Magen- und Duodenalgeschwüre und ulkusähnlichen 
Krankheitsbilder sowie der Fälle von Gallensteinerkrankungen in den letzten Jahren unstreitig zugenommen hat und daß auch die Zahl der Darmkranken in steigendem Wachstum begriffen war, und zwar teils im Zusammenhang mit den Nachwirkungen der vielen im Felde - und auch im Heimatgebiet - entstandenen Ruhr- und Paratyphuserkrankungen, teils unter dem Einflu $B$ der Tatsache, daß harmlose, klinisch latente chronische Kolitiden unter dem Einfluß der zellulosereichen Ernährung viel stärker als vorher in die Erscheinung traten. Wenn man in der Zeit, als das Kriegsbrot eingeführt wurde, gefürchtet hatte, daß die Mehrzahl der Gesunden das zellulosereiche Brot nicht vertragen könne, so wurden wir durch die praktische Erfahrung angenehm enttäuscht, indem diese gezeigt hat, daß bei der überwiegenden Mehrzahl der Menschen eine so weitgehende Anpassung an die neuen Ernährungsverhältnisse erfolgt ist, daß man geradezu daran denken konnte, 'daß sich der in der' Mitte zwischen Karnivoren und Herbivoren stehende Menschendarm allmählich den neuen Verhältnissen (eventuell durch Bereitstellung zelluloseverdauender Fermente?) nach der Richtung der Herbivorenleistung angepaßt hat. Indessen blieb trotzdem ein keineswegs kleiner Prozentsatz von Patienten übrig, bei welchen das Kriegsbrot dauernd $\mathrm{zu}$ höchst quälenden Empfindungen durch abnorme Gasbildung Anlaß gegeben hat. Das in früherer Zeit so oft erwähnte Krankheitsbild der ,Flatulenzdyspepsie“ hat unter dem Einfluß des Kriegsbrotes geradezu eine Wiedergeburt erfahren. Gaskoliken und Flatulenzschmerzen waren bei. vielen Menschen auch bei längerdauernder Zufuhr großer Mengen von Kohl und Rüben zu beobachten, doch hatte ich den Eindruck, als ob die in der Form der Flatulenzbeschwerden sich äußernde schädliche Wirkung des Brotes diejenige der Gemüse noch übertraf. Wirkten Kriegsbrot und grobe Gemüse bei empfindlichen Magenkranken - und die Zahl dieser war auch außerhalb der Gruppe wohlcharakterisierter Geschwürskranker nicht ganz klein - vorzugsweise auf mechanischem Wege, so war bei chronischen Kolitiden die Noxe mehr in unzuträglichen chemischen Zersetzungsprodukten $\mathrm{zu}$ suchen. Es war dabei der Notstand in bezug auf das Kriegsbrot um so größer, als es nicht immer gelang, denjenigen Patienten. welche tatsächlich des Weißbrotes dringend bedurften, solches behördlich $\mathrm{zu}$ verschaffen. Auch Zwiebacke und Teigwaren erzeugten, soweit sie nicht aus feinem Weizenmehl hergestellt waren, bei zahlreichen Verdauungskranken Beschwerden, und es zeigte sich gerade hier, wie außerordentlich empfindlich sich zahlreicheVerdauungskranke gegen die kleieartigenBestandteile der Zerealien erweisen. Sehr unangenehm machte sich in den zwei letzten Jahren vor allem auch der Mangel an R eis für zahlreiche au IDarmkatarrhen leidende Patienten bemerkbar. Für viele Fälle darf ein Gleiches auch beztiglich des Kaka 0 gesagt werden. Da bei der Knappheit an geeignetem Material die präparatorische Arbeit der Küche nicht völlig über die vorhandenen Schwierigkeiten Herr werden konnte, so haben sich nicht blo $B$ in privaten Haushalten, sondern auch im Krankenhausbetrieb auf dem vorliegenden Gebiete häufig sehr schwierige Situationen ergeben, und gar mancher Arzt war durch die Verhältnisse zu diätetischen Unterlassungen gezwungen, die er vor seinem Gewissen nicht verantworten kann. Dies gilt allerdings in höherem Grade für die Versorgung der Zivilbevölkerung als für die Lazarettverhältnisse, da die dort vorhandene bessere Belieferung es gestattete, die dringendsten Forderungen meistens in mehr oder weniger ausreichendem Grade zu erfüllen. Zum mindesten trifft dies für solche Sonderlazarette $\mathrm{zu}$, in welchen der Faktor Diätbehandlung eine besondere Berücksichtigung erfahren hat. Ich habe schon vor zwei Jahren an dieser Stelle (1917 Nr. 6) bei Besprechung der diätetischen Einrichtungen, die in einem größeren Reservelazarett für Verdauungs- und Stoffwechselkranke nach meinen Angaben getroffen worden sind, gezeigt, daß sich die modernen Forderungen der Diätetik durch geeignete Organisation auch im Lazarettbetrieb ohne größere Steigerung der Unkosten leicht erfüllen lassen. Daß aber eine Konzentration der einer speziellen Diät bedürftigen Kranken in Sonderlazarette tatsächlich notwendig war, ergab sich schon daraus, daß die diätetische Versorgung von Patienten, die einer Sonderernährung bedurften, in all- gemeinen Lazaretten aus technischen Gründen durch die Schwierigkeiten der Beschaffung und Herstellung der entsprechenden Speisen oft sehr erschwert war. Dazu kam mitunter auch noch der Umstand, daß nicht alle Aerzte auf dem Gebiete der Diätetik so geschult waren, daß sie unter schwierigen Verhăltnissen immer in der Lage waren, behelfsmäßige Einrichtungen in ausreichender Form zu treffen, und es soll deshalb mit Rücksicht auf die Kriegserfahrungen auch an dieser Stelle die Bemerkung nicht unterdrückt werden, daß für die Unterweisung vieler Aerzte in praktischer Diätetik nach gar mancher Richtung hin eine Besserung erwünscht erscheint. Für die Massenversorgung diätbedürftiger Kranker hat sich aber zuweilen auch noch ein weiterer Mangel bemerkbar gemacht, auf den ich gleichfalls schon vor vielen Jahren wiederholt hingewiesen habe, nämlich die Bereithaltung geeigneter küchentechnischer Kräfte. Denn die Herstellung einer geeigneten Diät ist nicht bloß eine Materialfrage, sondern auch eine technische Frage, und es ist ohne weiteres klar, daß bei entsprechendem Wissen der Aerzte und bei entsprechendem Können des Küchen- bzw. Pflegepersonals diätetische Improvisationen viel leichter gelingen als bei Fehlen der genannten Voraussetzungen. Aber auch unabhängig vom Krieg wäre es im Interesse unserer Krankenhäuser und Sanatorien sehr erwünscht, wenn sich auch auf diesem Gebiete eine Besserung vollziehen würde. ${ }^{1}$ )

Nehmen wir noch hinzu, daß die Verhältnisse des Krieges zu einer nicht geringen Zahl von Pilzvergiftungen, Trichinoseinfektionen $u$. ähnl. Anlaß gegeben haben, so haben uns die Erfahrungen über die Ernährung während des Krieges auch auf dem Gebiete der Krankenernährung gar manchen uns schon lange bekannten wichtigen Satz aufs neue in Erinnerung gebracht, gleichzeitig aber uns auch eine ganze Reihe von nicht so allgemein bekannten Hinweisen geliefert, die sowohl für die Behandlung des einzelnen Falles wie für gewisse organisatorische Maßnahmen beachtenswert erscheinen. Auch auf dem Gebiete der praktischen Diätetik wird die Zukunft von dem Grundsatz des „Sparen" beherrscht sein, und schon aus diesem Grunde wird es sich empfehlen, daß wir eine ganze Reihe von Lehren beherzigen, deren Kenntnis uns die Kriegsnot vermittelt hat. 